\title{
Two-step relaxation and the breakdown of the Stokes-Einstein relation in glass-forming liquids
}

\author{
Baicheng Mei $\odot,{ }^{1}$ Yuyuan Lu $\odot,{ }^{1, *}$ Lijia An, ${ }^{1, \dagger}$ and Zhen-Gang Wang ${ }^{2, *}$ \\ ${ }^{1}$ State Key Laboratory of Polymer Physics and Chemistry, Changchun Institute of Applied Chemistry, Chinese Academy of Sciences, \\ Changchun 130022, People's Republic of China \\ ${ }^{2}$ Division of Chemistry and Chemical Engineering, California Institute of Technology, Pasadena, California 91125, USA
}

(Received 30 August 2019; published 15 November 2019)

\begin{abstract}
It is well known that glass-forming liquids exhibit a number of anomalous dynamical phenomena, most notably a two-step relaxation in the self-intermediate scattering function and the breakdown of the StokesEinstein (SE) relation, as they are cooled toward the glass transition temperature. While these phenomena are generally ascribed to dynamic heterogeneity, specifically to the presence of slow- and fast-moving particles, a quantitative elucidation of the two-step relaxation and the violation of the SE relation in terms of these concepts has not been successful. In this work, we propose a classification of particles according to the rank order of their displacements (from an arbitrarily defined origin of time), and we divide the particles into long-distance (LD), medium-distance, and short-distance (SD) traveling particle groups. Using molecular-dynamics simulation data of the Kob-Andersen model, we show quantitatively that the LD group is responsible for the fast relaxation in the two-step relaxation process in the intermediate scattering function, while the SD group gives rise to the slow $(\alpha)$ relaxation. Furthermore, our analysis reveals that $\tau_{\alpha}$ is controlled by the SD group, while the ensemble-averaged diffusion coefficient $D$ is controlled by both the LD and SD groups. The combination of these two features provides a natural explanation for the breakdown in the SE relation at low temperature. In addition, we find that the $\alpha$-relaxation time, $\tau_{\alpha}$, of the overall system is related to the relaxation time of the LD particles, $\tau_{\mathrm{LD}}$, as $\tau_{\alpha}=\tau_{0} \exp \left(\Omega \tau_{\mathrm{LD}} / k_{\mathrm{B}} T\right)$.
\end{abstract}

DOI: $10.1103 /$ PhysRevE.100.052607

\section{INTRODUCTION}

Glass-forming liquids exhibit a number of anomalous dynamical phenomena as the temperature is cooled toward the glass-transition temperature [1-4]. For example, the selfintermediate scattering function $F_{\mathrm{s}}(\mathbf{q}, t)$, the self-part of the relaxation of the density-density correlation function, shows a distinct two-step relaxation-a fast relaxation followed by a plateau due to the cage effect before the final nonexponential decay in the form of the Kohlrausch-Williams-Watts (KWW) function $[5,6]$. Such a two-step relaxation has been loosely attributed to the presence of dynamic heterogeneity $[2,6,7]$ in glass-forming liquids at low temperatures, i.e., the presence of numerous heterogeneous regions of mesoscopic sizes, with some regions comprising fast-moving particles having fluidlike characteristics with low viscosity, and other regions made up of slow-moving particles exhibiting highly viscous characteristics. However, application of these concepts to $F_{\mathrm{s}}(\mathbf{q}, t)$ has not established a clear correlation between these two kinds of particles on the one hand, and the two kinds of relaxations in this function on the other. For example, an $F_{\mathrm{S}}(\mathbf{q}, t)$ defined solely in terms of the fast or slow particles, which is expected to show a simple one-step relaxation, still exhibits a two-step relaxation $[5,8]$.

\footnotetext{
*yylu@ ciac.ac.cn

$\dagger$ ijan@ciac.ac.cn

${ }^{\ddagger}$ zgw@caltech.edu
}

Another anomalous behavior in the glass-forming liquids is the breakdown of the Stokes-Einstein (SE) relation, which relates the self-diffusion coefficient $D$ to the viscosity or equivalently to the relaxation time $\tau_{\alpha}$ [9-12]. While for normal liquids the product $D \tau_{\alpha}$ is temperature-independent, numerous studies [13-19] in recent decades have shown that this relation is violated in glass-forming liquids at low temperatures. Conceptually, Hodgdon and Stillinger [20,21] associated this violation with dynamic heterogeneity; however, there is considerable debate as to the mechanistic explanation. It has been argued that slow particles determine the relaxation time $\tau_{\alpha}$, whereas the average diffusion coefficient $D$ is dominated by the fast particles [4]. A similar view [11,15] is that the slow and fast particles obey the SE relation separately, but the combined effect leads to a breakdown in the SE relation from the standpoint of the average relaxation time and diffusion coefficient [6]. However, some simulation studies find that the SE relation only holds for the slow particles and does not hold for the fast particles [22,23], while other studies suggest that the SE relation is violated for both the fast and slow particles $[24,25]$.

In this work, we investigate the origin of the two-step relaxation and the breakdown of the SE relation by conducting molecular-dynamics simulations of the classical KobAndersen model [26-28] consisting of a binary LennardJones (LJ) glass-forming liquid. Different from the slow- and fast-moving particles invoked in the literature, we propose an alternative classification according to the rank order of the particles measured by their observed displacements at any given time, where the small proportion of particles with 


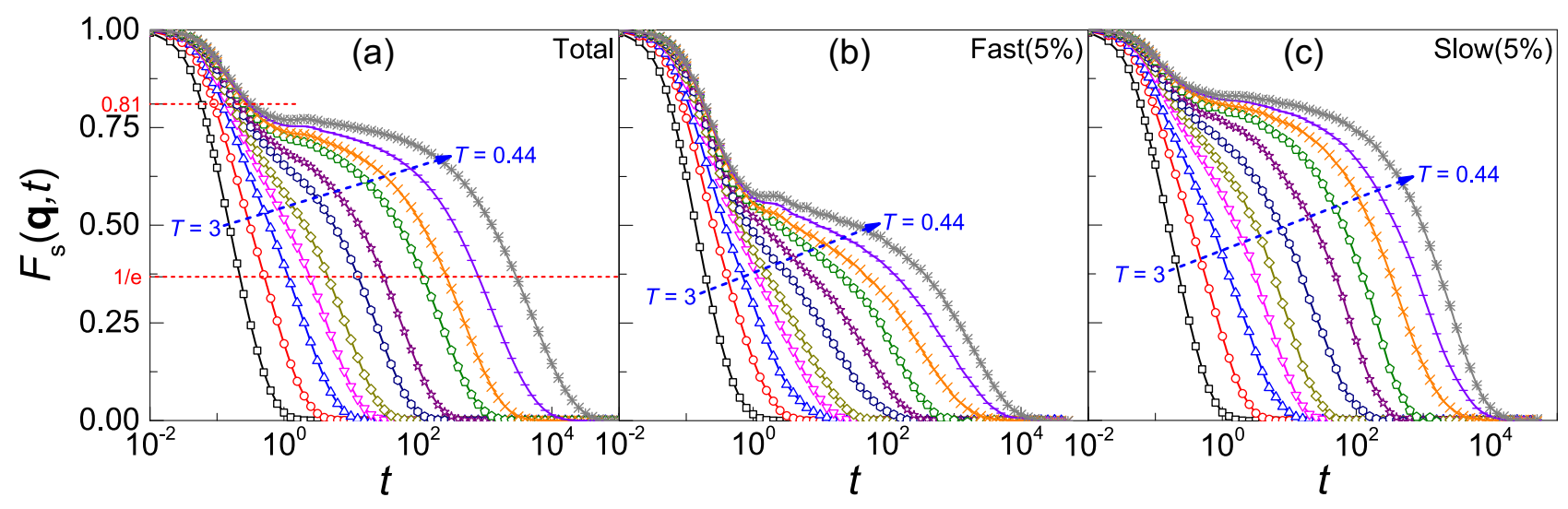

FIG. 1. The self-intermediate scattering function for all particles (a), and particles in LD (b) and SD (c) groups, at a series of temperatures. From left to right, the temperatures are $3.0,1.5,1.0,0.8,0.7,0.6,0.55,0.5,0.48,0.46$, and 0.44 , respectively. The red horizontal lines define the corresponding structural relaxation times.

the largest displacements is denoted as a long-distance (LD) traveler group, and those with the smallest displacements are denoted as a short-distance (SD) traveler group, while the remainder are denoted as a medium-distance (MD) traveler group. We find that the fast and slow relaxations in the self-intermediate scattering function can be unambiguously associated with the dynamics of the LD and SD groups, respectively. The relaxation times of the LD and SD groups, together with their diffusion coefficients, allow a straightforward elucidation of the mechanism of the breakdown in the SE relation at low temperatures. Further analysis reveals that there exists a strong correlation between different relaxation times: both the relaxation time for the SD group and the system relaxation ( $\alpha$-relaxation) times depend exponentially on the relaxation time of the LD group, implying that the $\alpha$ relaxation originates from multiple short-time rearrangement events. Finally, we present evidence for nonergodicity that persists to several orders of the system relaxation time.

\section{MODEL AND METHODS}

We performed molecular-dynamics simulation of the classical Kob-Anderson model [26-28] for a binary LennardJones mixture of particles A and B, which can mimic an amorphous metal or metalloid alloy $\mathrm{Ni}_{80} \mathrm{P}_{20}$ [29]. The interaction potential is given by

$$
V_{\alpha \beta}(r)=4 \epsilon_{\alpha \beta}\left[\left(\frac{\sigma_{\alpha \beta}}{r}\right)^{12}-\left(\frac{\sigma_{\alpha \beta}}{r}\right)^{6}\right],
$$

where $\alpha, \beta \in[\mathrm{A}, \mathrm{B}], \epsilon_{\alpha \beta}$ represents the depth of the potential well, $r$ is the distance between particles, and $\sigma_{\alpha \beta}$ is the value of $r$ for which $V_{\alpha \beta}$ is zero. All quantities are presented in reduced units, with the lengths given in units of $\sigma_{\mathrm{AA}}$, temperature in units of $\epsilon_{\mathrm{AA}}$ over the Boltzmann constant $k_{\mathrm{B}}$, and time in units of $\sqrt{m \sigma_{\mathrm{AA}}^{2} / \epsilon_{\mathrm{AA}}}$. The mass of each particle is assumed to be the same, $m=1.0$. Other $\mathrm{LJ}$ parameters are $\sigma_{\mathrm{AB}}=0.8 \sigma_{\mathrm{AA}}$, $\sigma_{\mathrm{BB}}=0.88 \sigma_{\mathrm{AA}}, \epsilon_{\mathrm{AB}}=1.5 \epsilon_{\mathrm{AA}}$, and $\epsilon_{\mathrm{BB}}=0.5 \epsilon_{\mathrm{AA}}$. These parameters are identical to those used by previous researchers $[27,28]$. We employ a cutoff distance of $r_{\mathrm{c}}=2.5$, beyond which $V_{\alpha \beta}$ is assumed to be zero. The number of particles $N$ is 1080 with $80 \%$ (864) A and 20\% (216) B. A cubic box with size $L_{\mathrm{x}}=L_{\mathrm{y}}=L_{\mathrm{z}}=9.655$ is used, corresponding to a number density $\rho$ of 1.2. For this system, the mode-coupling transition temperature is $T_{\mathrm{c}}=0.435[28,30,31]$. The simulations were conducted in the $N V T$ ensemble, and periodic boundary conditions were applied in all three directions. The equations of motion were integrated using the velocity form of the Verlet algorithm with a time step of 0.0025 . The system was equilibrated for at least $10 \tau_{\alpha}$ prior to the collection of statistical data. Simulations were performed for a series of temperatures starting at the highest $T=3.0$ down to $T=$ 0.42 .

\section{RESULTS}

\section{A. Decomposition of the self-intermediate scattering function}

In Fig. 1(a), we present the time evolution of the self-intermediate scattering function for the entire system, $F_{\mathrm{s}}(\mathbf{q}, t)=\left\langle\frac{1}{N} \sum_{j=1}^{N} \exp \left\{i \mathbf{q} \cdot\left[\mathbf{r}_{j}\left(t+t_{0}\right)-\mathbf{r}_{j}\left(t_{0}\right)\right]\right\}\right\rangle$, with $|\mathbf{q}|$ set to correspond to the first peak of the static structure factor, and $\langle\cdots\rangle$ represents the thermal average, which is obtained by averaging over both different $t_{0}$ and different samples. At high temperatures, $F_{\mathrm{s}}(\mathbf{q}, t)$ exhibits a single-step fast relaxation. With decreasing $T$, a shoulder appears at intermediate times, which reflects the caged motion of particles in glassy systems and divides the structural relaxation into two contributions representative of a two-step relaxation, where the primary peak corresponds to the short-time rapid relaxation and the shoulder corresponds to the long-time $\alpha$-relaxation [6]. Note that the $\alpha$-relaxation time $\tau_{\alpha}$ is defined as the time required for $F_{\mathrm{s}}(\mathbf{q}, t)$ to decay to $1 / e$.

In the literature, the fast- and slow-moving particles are defined by the square of their displacement, $\mathbf{r}_{i}^{2}\left(\tau^{*}\right)=\left[\mathbf{r}_{i}\left(t_{0}+\right.\right.$ $\left.\left.\tau^{*}\right)-\mathbf{r}_{i}\left(t_{0}\right)\right]^{2}$, over some characteristic time $\tau^{*}[15,32]$, where $t_{0}$ is an arbitrarily chosen origin of time. A particular particle $i$ will be defined as a fast- or slow-moving particle, respectively, if $\mathbf{r}_{i}^{2}\left(\tau^{*}\right)$ belongs to the top or bottom few percent, say $5 \%$, of all particles. $\tau^{*}$ is usually selected as the time $t^{*}$ when the corresponding non-Gaussian parameter is at its maximum $[5,8,33]$, which is slightly shorter than $\tau_{\alpha}$. Instead of the self-intermediate scattering function for all the particles, we define $F_{\mathrm{s}}(\mathbf{q}, t)$ for particles belonging only to the fast- and slow-moving particle groups, respectively. 


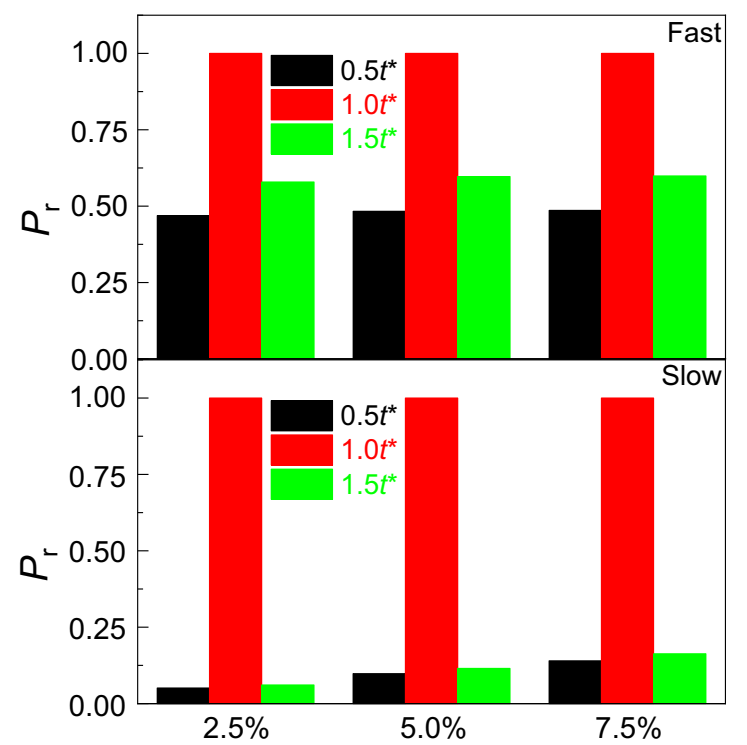

FIG. 2. Upper panel: fast-moving particles; lower panel: slowmoving particles. The histograms show the fraction of fast- and slowmoving particles defined at $t=t *$ that remain in the same respective groups at earlier $\left(0.5 t^{*}\right.$, black) and later $\left(1.5 t^{*}\right.$, green $)$ times. $2.5 \%$, $5 \%$, and $7.5 \%$ are the different thresholds used to define the fast- and slow-moving groups.

This is done by restricting the summation in the definition of $F_{\mathrm{s}}(\mathbf{q}, t)$ to include only particles in the fast- or slow-moving particle groups. We observe in Figs. 1(b) and 1(c) that both groups exhibit similar two-step relaxation behaviors to that for the entire particle set at low temperatures. Note that in this classification scheme, the motion state of a particle (i.e., whether a particle is slow- or fast-moving) was permanently assigned to a specific particle index $i$ according to its displacement at $t=t^{*}$, i.e., the identities of the particles in the fast- and slow-moving groups do not change with time. Thus, when examining the self-intermediate scattering function or the diffusion, any given particle carries the same state label, even though a fast particle in the top 5\% measured by its displacement at $t=t^{*}$ may not belong to the top 5\% of particles with the largest displacements at earlier or later times. Indeed, at $t=0.5 t^{*}$, only $48.3 \%$ of the fast particles defined using $t=t^{*}$ were actually fast, while at $t=1.5 t^{*}$, the fraction is $59.7 \%$. Even fewer overlaps are observed for the slow particles; see the lower panel in Fig. 2. In other words, the fastand slow-moving particles defined by their displacements at $t=\tau^{*}$ do not inform their actual displacements at earlier or later times.

We propose a classification of particles according to their displacements at any given time $t$ (measured from $t_{0}=0$ ), and we divide the particles into $\mathrm{LD}, \mathrm{MD}$, and $\mathrm{SD}$ groups based on the rank order of their displacements. At any given moment, the small fraction of particles (e.g., 5\%) with the largest displacements is considered to form the LD group, and those (e.g., 5\%) with the smallest displacements are assigned to the SD group, while the remainder $(90 \%)$ are considered to belong to the MD group. We have also used threshold values of $2.5 \%$ and $7.5 \%$ in ranking the particles; the results only show small quantitative differences. For clarity, we focus
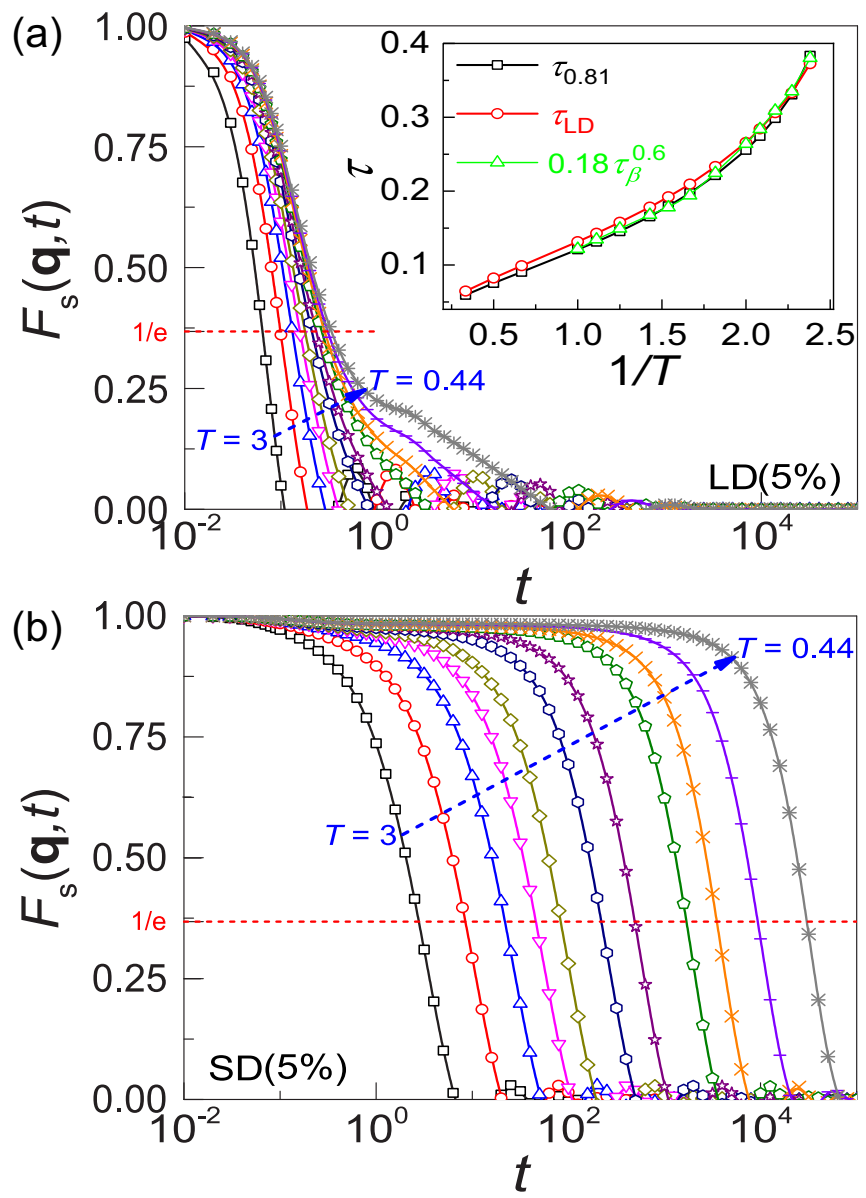

FIG. 3. The self-intermediate scattering function for (a) the LD traveling group (the top 5\% of total particles) and (b) the SD traveling group (the bottom $5 \%$ of total particles) at a series of temperatures. The red horizontal lines define the corresponding structural relaxation times. The inset in (a) displays the temperature dependence of the relaxation times corresponding to the LD group, the time required for the $F_{\mathrm{S}}(\mathbf{q}, t)$ of all particles to decay to $0.81\left(\tau_{0.81}\right)$, and $0.18 \tau_{\beta}^{0.6}$, where $\tau_{\beta}$ is the $\beta$-relaxation time, defined using the inflection in the $\log -\log$ plot of the mean squared displacement (MSD) of a particle system vs time [34].

on results obtained using the $5 \%$ threshold. Note that in this definition, the particle index is unimportant, i.e., a randomly chosen particle $i$ can change between the LD, MD, and SD groups with the evolution of time, but at each given time, the assignment of $\mathrm{LD}, \mathrm{SD}$, and MD will always be based on the rank order of the particles according to their displacements, regardless of the particle index. We now demonstrate that this classification leads to a natural explanation of the two-step relaxation process and the breakdown of the SE relation.

In Figs. 3(a) and 3(b), we present these restricted selfintermediate scattering functions for the LD and SD groups over the same range of $T$. Rather than the two-step relaxation behavior obtained for all particles, we observe that the $F_{\mathrm{s}}(\mathbf{q}, t)$ values for both $\mathrm{LD}$ and $\mathrm{SD}$ groups exhibit single-step relaxation behaviors, each with their own relaxation times, $\tau_{\mathrm{LD}}$ and $\tau_{\mathrm{SD}}$. If we normalize the time in Figs. 3(a) and 3(b) using their corresponding relaxation times, we obtain the results shown in Figs. 4(a) and 4(b). We find that the normalized curves 


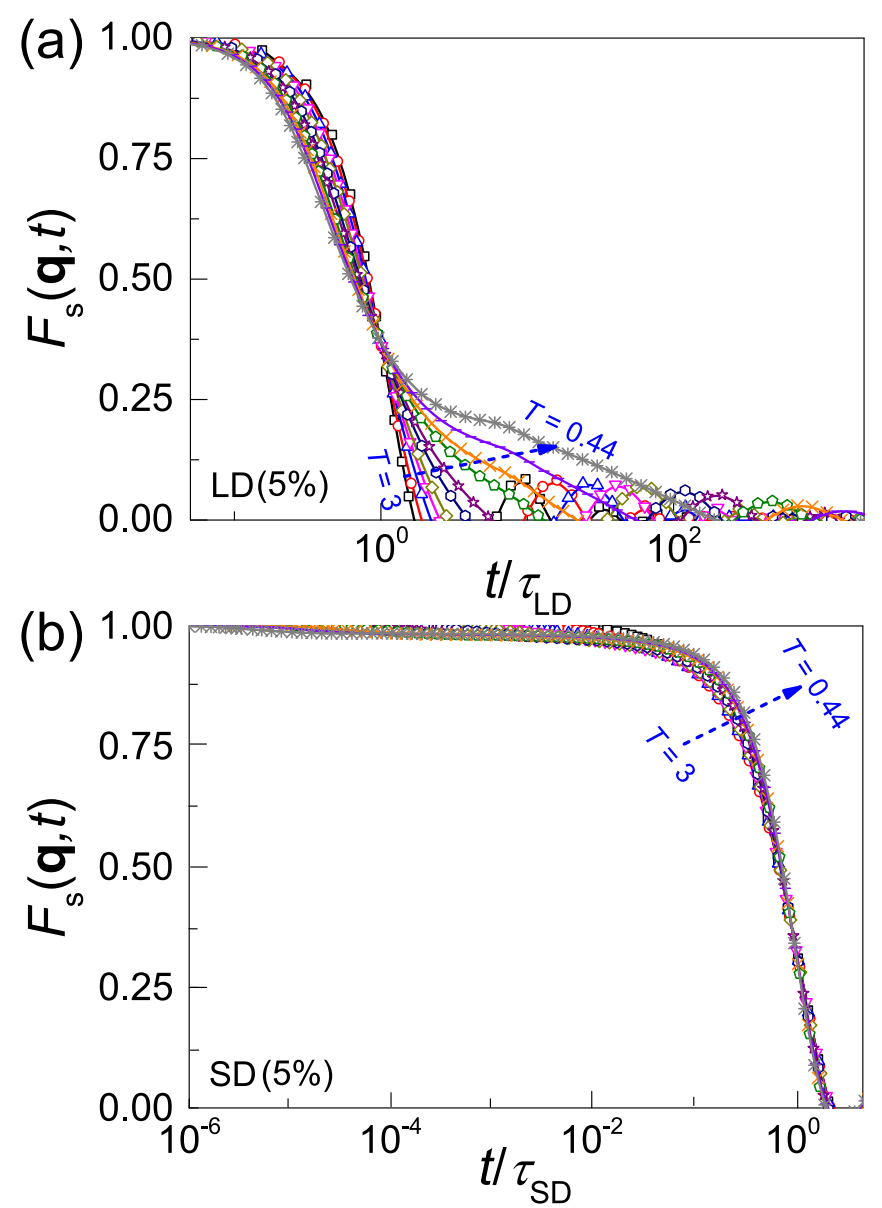

FIG. 4. The values of the self-intermediate scattering function for the LD group (a) and the SD group (b) with $t$ normalized by the corresponding relaxation time at a series of temperatures.

for the respective LD and SD groups approximately coincide, indicating that the LD and SD groups each exhibit similar relaxation behavior at different temperatures. The differences at long times observed in Fig. 4(a) are due to particle exchanges; see the discussions in Sec. III E.

The relaxation time of the SD group $\tau_{\mathrm{SD}}$ is found to be comparable to the overall relaxation time $\tau_{\alpha}$, but is far greater than that of the LD group $\tau_{\mathrm{LD}}$. We can introduce another characteristic time $\tau_{0.81}$ for all particles, which is defined as the time required for $F_{\mathrm{s}}(\mathbf{q}, t)$ to decay to 0.81 ; see Fig. 1(a). As is clear from the inset of Fig. 3(a), $\tau_{0.81}$ is approximately equal to $\tau_{\mathrm{LD}}$ at all temperatures considered. This analysis demonstrates a clear correspondence between the rapid relaxation with the dynamics of the LD group, and the slow relaxation with the SD group.

\section{B. Temperature dependence of time scales}

Figure 5 shows the temperature dependence of the different relaxation times. It is clear that $\tau_{\mathrm{LD}}$ exhibits Arrhenius behavior, with a small slope in the $1 / T$ plot, suggesting a low activation barrier. $\tau_{\mathrm{SD}}$, on the other hand, shows clearly superArrhenius behavior; the curves can be well fitted by the classical Vogel-Fulcher-Tammann equation $\tau=\tau_{0} \exp \left[B T_{0} /(T-\right.$ $T_{0}$ )] [35-37], where $B$ and $T_{0}$ are fitting parameters, and $\tau_{0}$

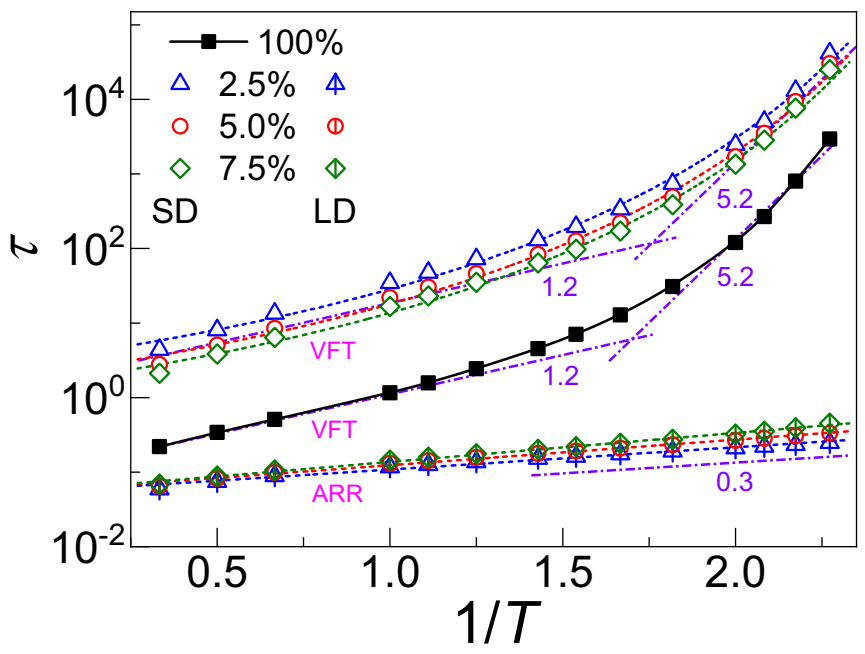

FIG. 5. Temperature dependence of the relaxation times for all particles, the LD group (based on the $2.5 \%, 5 \%$, or $7.5 \%$ thresholds), and the SD group (based on the $2.5 \%, 5 \%$, or $7.5 \%$ thresholds).

represents the relaxation time in the high-temperature limit. Moreover, the temperature dependence of $\tau_{\mathrm{SD}}$ is similar to that of the system relaxation time $\tau_{\alpha}$. In particular, the slopes of both relaxation times with respect to $1 / T$ in the semilog plot in the Arrhenius regime are identical $(\approx 1.2)$, as are the limiting slopes in the super-Arrhenius regime $(\approx 5.2)$. Note that the limiting slopes in the super-Arrhenius regime are indicated for convenience of discussion and do not imply the existence of a linear dependence in the logarithm of the relaxation time on $1 / T$.

The Arrhenius temperature dependence in $\tau_{\mathrm{LD}}$ implies a simple activated process. To demonstrate this, we plot the probability distribution of $r$ [i.e., $P(r)$ ] for all particles at $t=\tau_{\mathrm{LD}}$ in Fig. 6(a). We observe that the population of LD particles are those that have undergone displacements exceeding half the particle radius $r \approx 0.24$. If we consider this as a threshold value for a particle to escape its local cage, we can identify $\tau_{\mathrm{LD}}$ as the timescale for this activated event. This reasoning is corroborated by applying the nonlinear Langevin equation (NLE) theory of Schweizer and co-workers [38-40], which provides the following $r$-dependent dynamic free energy $F_{\text {dyn }}(r)$ for a tagged particle:

$$
\begin{aligned}
\beta F_{\text {dyn }}(r)= & -3 \ln (r) \\
& -\int \frac{d \mathbf{q}}{(2 \pi)^{3}} \frac{\rho C^{2}(q) S(q)}{1+S^{-1}(q)} e^{-\frac{(q r)^{2}}{6}\left[1+S^{-1}(q)\right]} .
\end{aligned}
$$

Here, $\beta=1 / k_{\mathrm{B}} T, \rho$ is the number density of the particles, $C(q)$ is the direct correlation function [41,42], and $S(q)=[1-\rho C(q)]^{-1}$ is the structure factor, where $q=|\mathbf{q}|$ is the wave number. We employed Eq. (2) to obtain the $r$-dependence of $\beta F_{\text {dyn }}(r)$ for our LJ system at different temperatures using the $S(q)$ obtained from our simulation. The results are shown in Fig. 6(b). For low temperatures $(T<1.0), \beta F_{\mathrm{dyn}}(r)$ acquires a minimum (transient localized state) at $r<r_{\mathrm{B}}$ and an energy barrier at $r_{\mathrm{B}}$. This energy barrier indicates the emergence of hopping transport (i.e., activated rearrangement). Importantly, we find $r_{\mathrm{B}} \approx 0.24$, 

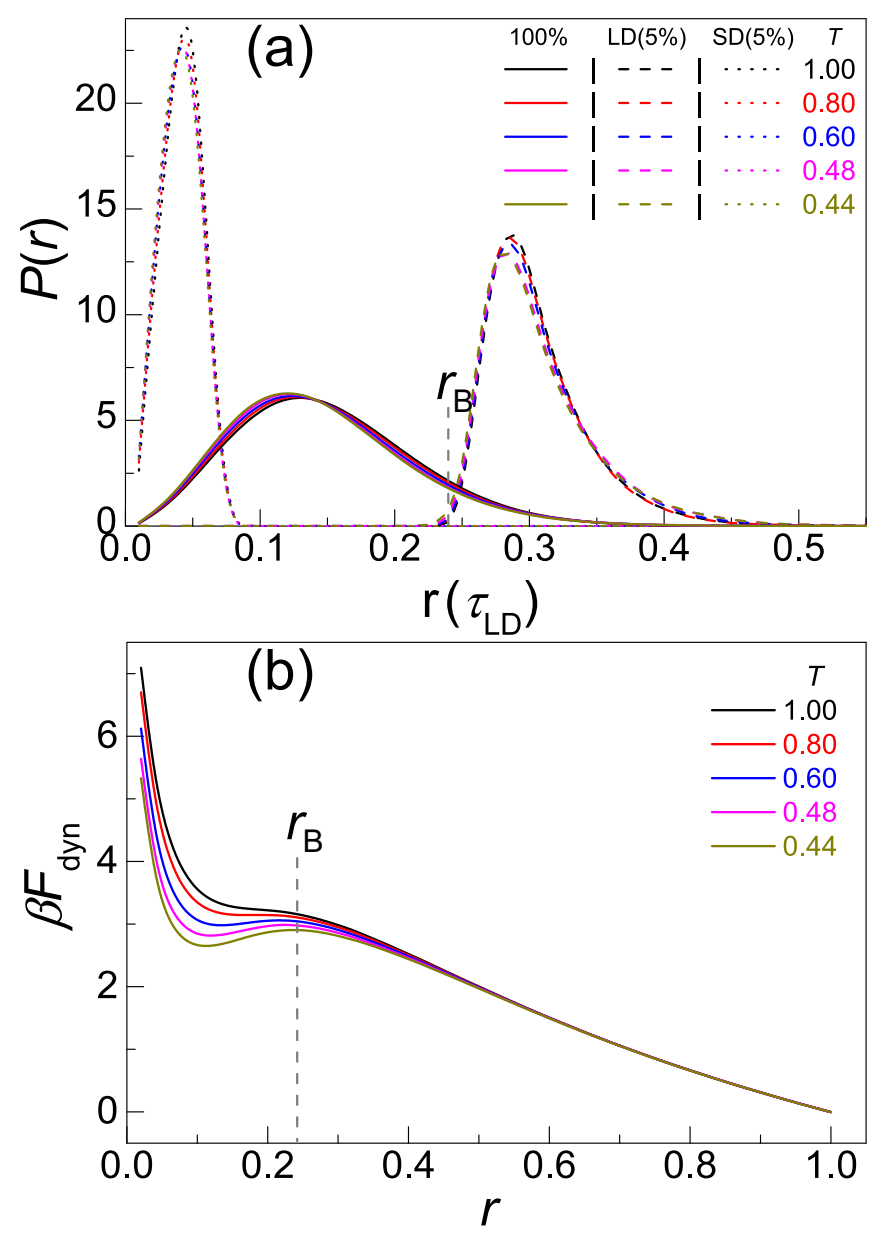

FIG. 6. (a) Probability distribution of particle displacement $r$ for all particles, and particles in LD and SD groups, at a series of temperatures. (b) Nonlinear Langevin equation dynamic free-energy barriers as a function of $r$ at a series of temperatures.

which coincides with the threshold value shown in Fig. 6(a). For comparison, we also show in the same figure the displacement distribution for the SD particles; clearly their displacements are far below $r_{\mathrm{B}} \approx 0.24$. These results are inconsistent with the conventional picture of all the particles performing localized motion within their cages as being responsible for the faster relaxation mode [6].

\section{Relationship between different timescales}

Fluctuations in the density correlation functions of glassforming liquids typically approach a plateau value after undergoing an initial rapid relaxation. This relaxation is known as the $\beta$-relaxation process [43]. While recent studies [34] have defined $\tau_{\beta}$ using the inflection in the log-log plot of the meansquared displacement (MSD) of a particle system versus time, hereafter referred to as $\tau_{\beta}$ (MSD), our analysis based on the LD and SD classification suggests a similarity between the relaxation of the LD particles and the $\beta$ relaxation. This implies that the $\beta$-relaxation process may originate from the activated relaxation of some of the particles.

The conventional understanding of $\beta$ - and $\alpha$-relaxation is that these are two different relaxation modes for the system that occur on different timescales. However, a recent study has shown that $\tau_{\beta}$ (MSD) scales with the dynamic heterogeneity length as $\tau_{\beta}(\mathrm{MSD}) \sim \xi^{0.8}$ [34]. We thus expect a similar relationship between $\tau_{\mathrm{LD}}$ and $\xi$, i.e., $\tau_{\mathrm{LD}} \sim \xi^{z}$. This relationship is shown in Fig. 7(a) with different criteria for defining the LD group. The exponent varies between 0.40 and 0.56 . Taking the $5 \%$ criterion, we get $z \approx 0.5$. On the other hand, according to the recent work of Das et al. [44], $\tau_{\alpha}$ satisfies the following relation with $\xi$ :

$$
\tau_{\alpha}=\tau_{0} \exp \left(\frac{\Omega \xi^{\gamma}}{k_{\mathrm{B}} T}\right),
$$

where $\Omega$ is a $T$-independent parameter and $\gamma$ is a universal exponent. This same study found that the average value of $\gamma$ for a three-dimensional system is approximately 0.5 , the same value as our exponent $z$ in scaling between $\tau_{\mathrm{LD}}$ and $\xi$. Therefore, we can substitute $\xi^{\gamma}$ in Eq. (3) with $\tau_{\mathrm{LD}}$, which yields the following relation:

$$
\tau_{\alpha}=\tau_{0} \exp \left(\frac{\Omega \tau_{\mathrm{LD}}}{k_{\mathrm{B}} T}\right) .
$$

This relation is plotted in Fig. 7(b), which indicates that Eq. (4) holds approximately over the full range of temperatures for all the different LD criteria. Moreover, a similar plot of Eq. (4) in Fig. 7(c), but with $\tau_{\alpha}$ replaced by $\tau_{\mathrm{LD}}$, demonstrates good agreement with this relation as well for all the temperatures in the supercooled regime. Combining $\tau_{\beta} \sim \xi^{0.8}$ and $\tau_{\mathrm{LD}} \sim \xi^{0.5}$, we also obtain a relation between the $\beta$-relaxation and the $\mathrm{LD}$ relaxation processes, i.e., $\tau_{\mathrm{LD}} \sim$ $\tau_{\beta}^{0.6}\left(\approx \tau_{\beta}^{5 / 8}\right)$, with a prefactor about 0.18 , as shown in the inset of Fig. 3(a). These results suggest that the $\beta$-relaxation process is directly controlled by the short-time activated rearrangements of particles in the LD group, whereas the long-time relaxation process results from multiple, successive activated rearrangements of these particles. Furthermore, an analysis of the spatial correlations of particles within the LD group on a timescale of $\tau_{\alpha}$ indicates that these particles tend to form stringlike structures, which is consistent with earlier observations of correlated stringlike motion of particles near the glass transition [5,33]. This indirectly supports the physical picture of the excitation chain [45-47] and string models [48-50] that stringlike structures are responsible for dynamic relaxation processes.

\section{Breakdown of the SE relation}

We now turn to the diffusion of the particles and the SE relation. In contrast to the behavior for the relaxation times, the values of $1 / D$ for all particles as well as for the separate LD and SD groups all exhibit significant super-Arrhenius behavior with respect to $1 / T$ at low temperatures; see Fig. 8(a). Moreover, the slopes of all curves for both groups agree well with the slopes obtained for all particles in the Arrhenius regime. However, in the super-Arrhenius regime the rate of change exhibits some minor differences between the SD and LD groups and all the particles, with the SD group having the largest slope, the LD group having the least, and all the particles intermediate between the SD and LD groups.

From Figs. 5 and 8(a), it is clear that the temperature dependence of $1 / D$ for the SD groups follows essentially that 

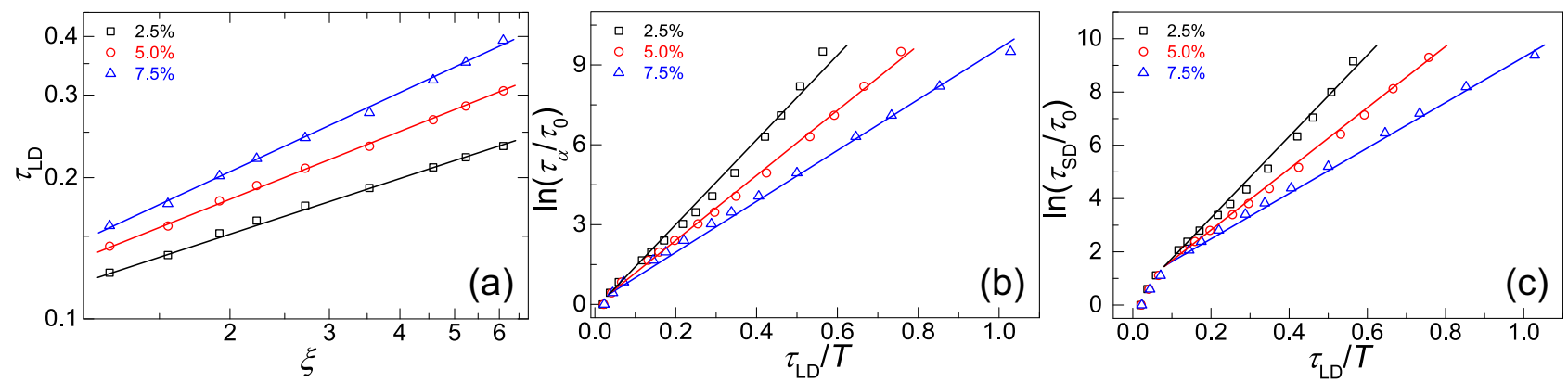

FIG. 7. (a) Relationship between $\tau_{\mathrm{LD}}$ and the dynamic heterogeneity length $\xi$ for the system with threshold values for the LD group. (b) Correlation between the relaxation time for the system $\tau_{\alpha}$ and $\tau_{\mathrm{LD}}$. (c) Correlation between $\tau_{\mathrm{SD}}$ and $\tau_{\mathrm{LD}}$.

of $\tau_{\mathrm{SD}}$, implying the $\mathrm{SE}$ relation for this group. It is also clear that the SE relation does not hold for the LD group since $\tau_{\mathrm{LD}}$ increases with $1 / T$ much slower than that of the other groups, even in the Arrhenius regime. When all particles are considered, the SE relation is obeyed in the Arrhenius regime, because both $\tau_{\alpha}$ and $1 / D$ follow the same temperature dependence. But in the super-Arrhenius regime, the slope in $1 / D$ is less than that in $\tau_{\alpha}$, so there is an upward turn in the product $D \tau_{\alpha}$, i.e., the $\mathrm{SE}$ relation is violated in this regime.

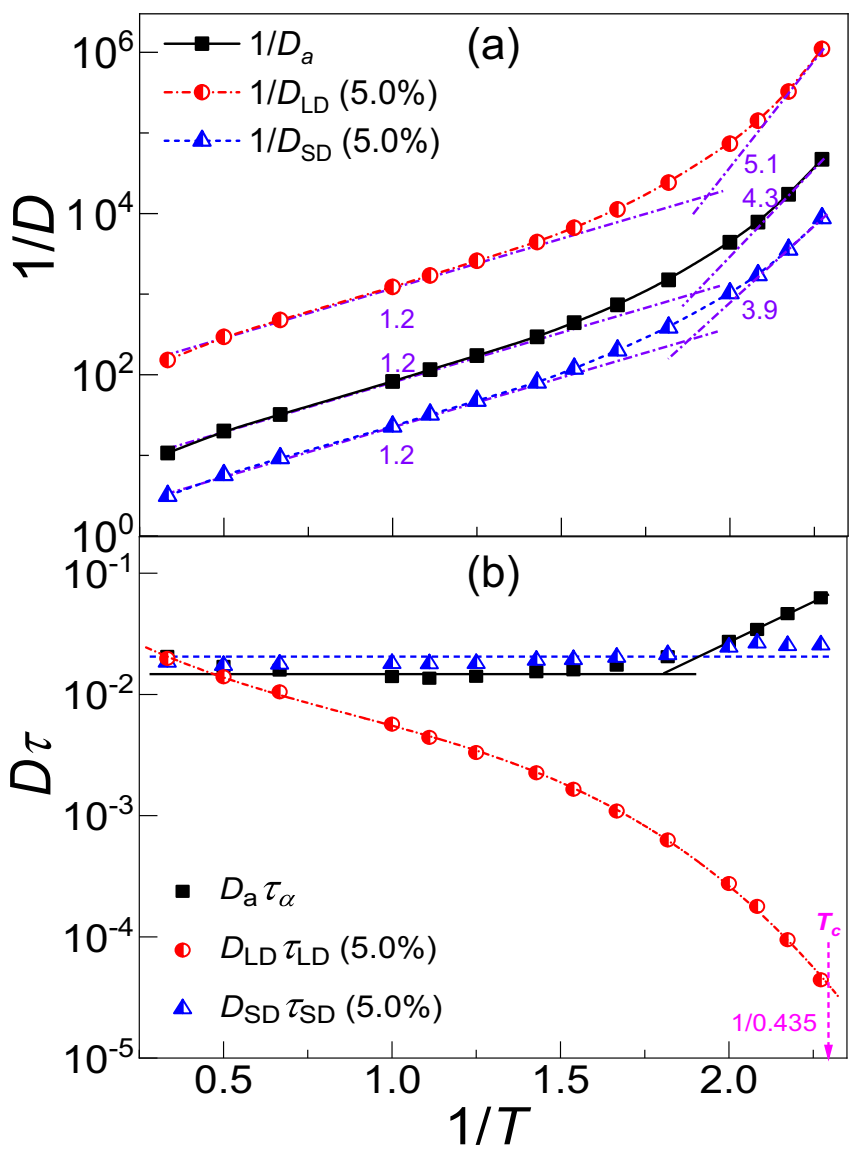

FIG. 8. Temperature dependence of (a) the reciprocal diffusion coefficient and (b) the product of the individual relaxation times $\tau$ and the corresponding diffusion coefficient $D$ for all particles, the LD group, and the SD group.
These behaviors are shown in Fig. 8(b), which presents the product $D \tau$ using $D$ and $\tau$ for each group.

\section{E. Memory effects and nonergodicity in LD and SD particles}

To gain insight into the relationship between the LD and SD particle groups introduced in this work and the fast- and slow-moving particles used in previous work, we return to the overlap fractions for these two groups of particles at different selected times shown in Fig. 2. While the figure demonstrates that the fast- and slow-moving particles are not permanentwhich is the main reason that they fail to explain the two-step relaxation in the self-intermediate scattering function-it also reveals that a significant fraction of the fast-moving particles (defined as the longest travelers at time $t^{*}$ ) is also in the LD group at $0.5 t^{*}$ and $1.5 t^{*}$, suggesting some memory effects. The fraction is less for the slow-moving particles, but still significantly higher than the corresponding threshold fraction that defines the group (for example, for the $5 \%$ threshold, the overlap is about $10 \%$ ).

To show this effect more quantitatively, in Fig. 9 we show the time evolution of the retention probability of particles in the LD or the SD group, $P_{\text {ret }}(t)=\left\langle N_{\mathrm{LD}-1}(t)\right\rangle / N_{\mathrm{LD}-0}$ or $\left\langle N_{\mathrm{SD}-1}(t)\right\rangle / N_{\mathrm{SD}-0}$. Here, $N_{\mathrm{LD}-0}\left(N_{\mathrm{SD}-0}\right)$ represents the number

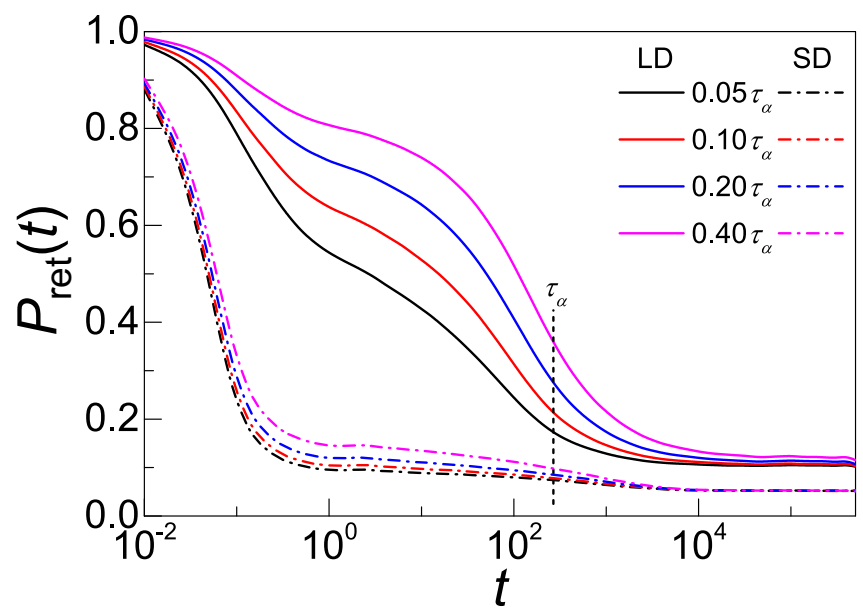

FIG. 9. Time evolution of the retention fraction $P_{\text {ret }}(t)$, i.e., the fraction of LD particles (solid line) and the SD particles (dash-dotted line) defined at time $t_{0}$ that remain in their respective groups at $t_{0}+$ $t$, for $T=0.48$. Four reference times $t_{0}$ are chosen: $0.05 \tau_{\alpha}, 0.1 \tau_{\alpha}$, $0.2 \tau_{\alpha}$, and $0.4 \tau_{\alpha}$. 


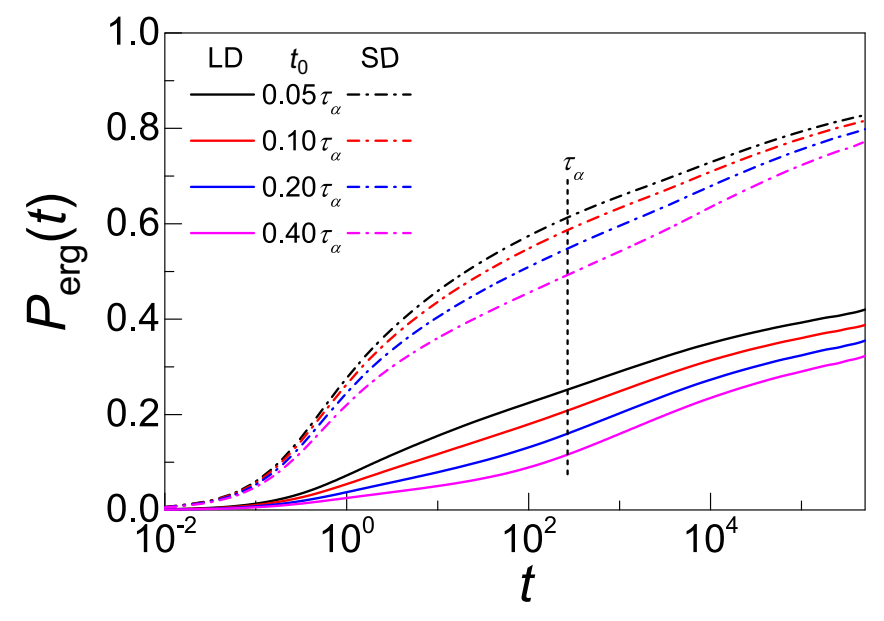

FIG. 10. Time evolution of $P_{\text {erg }}(t)$ at $T=0.48$ for the LD (solid lines) and the SD (dash-dotted lines) particles. Four reference times $t_{0}$ are chosen: $0.05 \tau_{\alpha}, 0.1 \tau_{\alpha}, 0.2 \tau_{\alpha}$, and $0.4 \tau_{\alpha}$.

of instantaneous LD (SD) particles at the reference time $t_{0}$ for defining the particle groups, and $\left\langle N_{\mathrm{LD}-1}(t)\right\rangle\left[\left\langle N_{\mathrm{SD}-1}(t)\right\rangle\right]$ is the average number of particles that remain in their respective group at $t+t_{0}$. On timescales of $\tau_{\alpha}, P_{\text {ret }}(t)$ has decayed considerably, suggesting that the identity of the particles within the LD (SD) group varies greatly. Note that the retention fraction is larger for the larger reference time, since the difference in the displacement between particles in the LD (SD) group and particles in other groups is larger, and hence it is less frequent that an LD (SD) particle will fall out of that group. On the other hand, $P_{\text {ret }}(t)$ remains appreciably greater than the threshold value employed for defining these groups $(5 \%)$ on the timescale of $\tau_{\alpha}$, suggesting that there is substantial overlap between the fast- and slow-moving particles in the literature (defined using a fixed time $\tau^{*}$ ) and our LD and SD particles on this time scale. These two results simultaneously explain why the conventional concept of fast- and slow-moving particles fails to offer a clear explanation of the two-step relaxation and the violation of SE relation, but nevertheless has proven useful in describing many classical phenomena in glass-forming liquids.

To further elucidate the memory effects in the LD and SD particles, we define the following function: $P_{\operatorname{erg}}(t)=$ $\left[\left\langle N_{\mathrm{LD}}^{*}(t)\right\rangle-N_{\mathrm{LD}-0}\right] /\left(N-N_{\mathrm{LD}-0}\right)$ or $\left[\left\langle N_{\mathrm{SD}}(t)\right\rangle-N_{\mathrm{SD}-0}\right] /(N-$ $\left.N_{\mathrm{SD}-0}\right)$. Here, $N$ is the total number of particles in the system, $N_{\text {LD-0 }}\left(N_{\mathrm{SD}-0}\right)$ is the same instantaneous number of LD (SD) particles defined for $P_{\mathrm{ret}}(t)$, and $N_{\mathrm{LD}}^{*}(t)\left[N_{\mathrm{SD}}^{*}(t)\right]$ is the average number of particles that have been in the LD (SD) group at least once during the period from $t_{0}$ to $t_{0}+t$. Clearly, $N_{\mathrm{LD}}^{*}(t=0)=N_{\mathrm{LD}-0}\left[N_{\mathrm{SD}}^{*}(t=0)=N_{\mathrm{SD}-0}\right]$, while for $t \rightarrow \infty$, $N_{\mathrm{LD}}^{*}=N_{\mathrm{SD}}^{*}=N$, because all the particles will have been in the LD (SD) group at least once if the system is ergodic. Since $\tau_{\alpha}$ is considered the longest relaxation time of the system, it is expected that the function $P_{\text {erg }}(t)$ should approach 1 on timescales of $\tau_{\alpha}$. Figure 10 shows the time evolutions of $P_{\text {erg }}(t)$ for different choices of $t_{0}$. Surprisingly, the values of $P_{\text {erg }}(t)$ are far less than 1 even for times greater than $10^{3} \tau_{\alpha}$. Therefore, judged by the behavior of this function, the relaxation time appears to be greater than $10^{3} \tau_{\alpha}$. In other words, on timescales of $10^{3} \tau_{\alpha}$, the system behaves nonergodic. In addition, we note from Fig. 10 that $P_{\mathrm{erg}}(t)$, especially for the SD particles, becomes substantial at $\tau_{\alpha}$, indicating that a significant fraction of the $\mathrm{LD}$ and $\mathrm{SD}$ particles have undergone exchanges. The fact that additional time even up to $10^{3} \tau_{\alpha}$ does not result in significantly more relaxation suggests that many of the exchanges are futile, i.e., they take place within the same particle group, thus confirming the long-term memory effects in these particle groups.

\section{CONCLUSIONS}

In summary, we have proposed a classification of the particles into $\mathrm{LD}, \mathrm{MD}$, and $\mathrm{SD}$ groups according to their observed displacements to characterize the dynamic heterogeneity in glass-forming liquids. With these newly defined particle groups, the two-step relaxation process in the intermediate scattering function $F_{\mathrm{s}}(\mathbf{q}, t)$ can be naturally assigned to the relaxation of the LD and SD groups, respectively, where the LD group contributes to the first-step (rapid) relaxation at short-time scales and the SD group determines the second-step $(\alpha)$-relaxation at long times. By analyzing the temperature dependence of the various relaxation times, we have discovered a strong exponential relationship between the relaxation time for the $\mathrm{LD}$ particles $\tau_{\mathrm{LD}}$ on the one hand, and the relaxation time for the SD particles $\tau_{\mathrm{SD}}$ and the $\alpha$-relaxation time $\tau_{\alpha}$ on the other. We believe that this exponential dependence implies that the long-time $\alpha$ relaxation results from multiple short-time rearrangement events. Moreover, while the value of $\tau_{\alpha}$ is controlled primarily by the SD particles, the diffusion coefficient is determined by both the LD and SD groups. Therefore, the breakdown of the SE relation at low temperatures can be understood as a direct consequence of the different contributions of the LD and SD groups to the relaxation time and the diffusion coefficient.

We have further analyzed the memory effects in the LD and SD particles by defining a retention probability and a measure of the ergodicity in the particle identity. Our results show that there is a substantial fraction of particles transitioning out of their respective groups on timescales of $\tau_{\alpha}$, yet a significant fraction remains, thus clarifying why the traditional concept of fast- and slow-moving particles fails to explain the two-step relaxation process and the breakdown of the SE relation, but is nevertheless useful for describing many dynamic phenomena in glass-forming liquids. Surprisingly, we find that the system behaves nonergodic up to three orders of magnitude of $\tau_{\alpha}$, judged by the ergodicity (or lack thereof) in the particle identity. We hope these results offer some fresh perspectives on the dynamic behavior in glass-forming liquids, and contribute to the development of new theories for describing glass dynamics.

\section{ACKNOWLEDGMENTS}

This work was supported by the Science Challenge Project (Grant No. TZ2018004), the National Natural Science Foundation of China (Grants No. 21674113 and No. 21790340), the Key Research Program of Frontier Sciences, CAS (Grant No. QYZDY-SSW-SLH027), the International Partnership Program of CAS (Grant No. 121522KYSB20160015), 
and Jilin Scientific and Technological Development Program (Grant No. 20180519001JH). Y.L. acknowledges the
Youth Innovation Promotion Association of CAS (Grant No. 2016204) for financial support.
[1] P. G. Debenedetti and F. H. Stillinger, Nature (London) 410, 259 (2001).

[2] L. Berthier and G. Biroli, Rev. Mod. Phys. 83, 587 (2011).

[3] B. Mei, Y. Lu, L. An, H. Li, and L. Wang, Phys. Rev. E 95, 050601(R) (2017).

[4] M. D. Ediger, Annu. Rev. Phys. Chem. 51, 99 (2000).

[5] C. Donati, S. C. Glotzer, P. H. Poole, W. Kob, and S. J. Plimpton, Phys. Rev. E 60, 3107 (1999).

[6] K. Binder and W. Kob, Glassy Materials and Disordered Solids: An Introduction to their Statistical Mechanics (World Scientific, Singapore, 2011).

[7] L. Berthier, Physics 4, 42 (2011).

[8] W. Kob, C. Donati, S. J. Plimpton, P. H. Poole, and S. C. Glotzer, Phys. Rev. Lett. 79, 2827 (1997).

[9] J. D. Eaves and D. R. Reichman, Proc. Natl. Acad. Sci. USA 106, 15171 (2009).

[10] P. Charbonneau, Y. Jin, G. Parisi, and F. Zamponi, Proc. Natl. Acad. Sci. USA 111, 15025 (2014).

[11] M. G. Mazza, N. Giovambattista, H. E. Stanley, and F. W. Starr, Phys. Rev. E 76, 031203 (2007).

[12] A. D. S. Parmar, S. Sengupta, and S. Sastry, Phys. Rev. Lett. 119, 056001 (2017).

[13] D. N. Perera and P. Harrowell, Phys. Rev. Lett. 81, 120 (1998).

[14] S.-H. Chen, F. Mallamace, C.-Y. Mou, M. Broccio, C. Corsaro, A. Faraone, and L. Liu, Proc. Natl. Acad. Sci. USA 103, 12974 (2006).

[15] P. Kumar, S. Buldyrev, S. Becker, P. Poole, F. W. Starr, and H. Stanley, Proc. Natl. Acad. Sci. USA 104, 9575 (2007).

[16] S.-H. Chong and W. Kob, Phys. Rev. Lett. 102, 025702 (2009).

[17] S. Sengupta, S. Karmakar, C. Dasgupta, and S. Sastry, J. Chem. Phys. 138, 12A548 (2013).

[18] L. Xu, F. Mallamace, Z. Yan, F. W. Starr, S. V. Buldyrev, and H. E. Stanley, Nat. Phys. 5, 565 (2009).

[19] S. Pan, Z. W. Wu, W. H. Wang, M. Z. Li, and L. Xu, Sci. Rep. 7, 39938 (2017).

[20] J. A. Hodgdon and F. H. Stillinger, Phys. Rev. E 48, 207 (1993).

[21] F. H. Stillinger and J. A. Hodgdon, Phys. Rev. E 50, 2064 (1994).

[22] S. K. Kumar, G. Szamel, and J. F. Douglas, J. Chem. Phys. 124, 214501 (2006).

[23] S. Sengupta and S. Karmakar, J. Chem. Phys. 140, 224505 (2014).

[24] M. G. Mazza, N. Giovambattista, F. W. Starr, and H. E. Stanley, Phys. Rev. Lett. 96, 057803 (2006).
[25] S. R. Becker, P. H. Poole, and F. W. Starr, Phys. Rev. Lett. 97, 055901 (2006).

[26] W. Kob and H. C. Andersen, Phys. Rev. Lett. 73, 1376 (1994).

[27] S. Sastry, P. G. Debenedetti, and F. H. Stillinger, Nature (London) 393, 554 (1998).

[28] B. Mei, Z. Wang, Y. Lu, H. Li, and L. An, J. Chem. Phys. 147, 114507 (2017).

[29] T. A. Weber and F. H. Stillinger, Phys. Rev. B 31, 1954 (1985).

[30] L. Berthier, G. Biroli, D. Coslovich, W. Kob, and C. Toninelli, Phys. Rev. E 86, 031502 (2012).

[31] W. Kob and H. C. Andersen, Phys. Rev. E 52, 4134 (1995).

[32] A. S. Keys, L. O. Hedges, J. P. Garrahan, S. C. Glotzer, and D. Chandler, Phys. Rev. X 1, 021013 (2011).

[33] C. Donati, J. F. Douglas, W. Kob, S. J. Plimpton, P. H. Poole, and S. C. Glotzer, Phys. Rev. Lett. 80, 2338 (1998).

[34] S. Karmakar, C. Dasgupta, and S. Sastry, Phys. Rev. Lett. 116, 085701 (2016).

[35] H. Vogel, Phys. Z. 22, 645 (1921).

[36] G. S. Fulcher, J. Am. Ceram. Soc. 8, 339 (1925).

[37] G. Tammann and W. Hesse, Z. Anorg. Allg. Chem. 156, 245 (1926).

[38] K. S. Schweizer and E. J. Saltzman, J. Chem. Phys. 119, 1181 (2003).

[39] E. J. Saltzman and K. S. Schweizer, J. Chem. Phys. 119, 1197 (2003).

[40] K. S. Schweizer, J. Chem. Phys. 123, 244501 (2005).

[41] S. Mirigian and K. S. Schweizer, J. Phys. Chem. Lett. 4, 3648 (2013).

[42] J.-P. Hansen and I. R. McDonald, Theory of Simple Liquids (Academic, London, 2006).

[43] H. B. Yu, W. H. Wang, H. Y. Bai, and K. Samwer, Natl. Sci. Rev. 1, 429 (2014).

[44] R. Das, I. Tah, and S. Karmakar, J. Chem. Phys. 149, 024501 (2018).

[45] J. S. Langer and A. Lemaitre, Phys. Rev. Lett. 94, 175701 (2005).

[46] J. S. Langer, Phys. Rev. E 73, 041504 (2006).

[47] J. S. Langer, Phys. Rev. Lett. 97, 115704 (2006).

[48] B. A. P. Betancourt, J. F. Douglas, and F. W. Starr, J. Chem. Phys. 140, 204509 (2014).

[49] P. Z. Hanakata, J. F. Douglas, and F. W. Starr, Nat. Commun. 5, 4163 (2014).

[50] B. A. P. Betancourt, P. Z. Hanakata, F. W. Starr, and J. F. Douglas, Proc. Natl. Acad. Sci. USA 112, 2966 (2015). 